\title{
Gut Dysfunction and Non-alcoholic Fatty Liver Disease
}

\author{
Felix Grabherr, Christoph Grander, Maria Effenberger, Timon Erik Adolph and Herbert Tilg* \\ Department of Internal Medicine I, Gastroenterology, Hepatology, Metabolism and Endocrinology, University Hospital \\ Innsbruck, Innsbruck, Austria
}

OPEN ACCESS

Edited by:

Pierre De Meyts,

Duve Institute, Catholic University of

Louvain, Belgium

Reviewed by:

Anne-Francoise Burnol,

Institut National de la Santé et de la

Recherche Médicale

(INSERM), France

Heike M. Hermanns,

Universitätsklinikum

Würzburg, Germany

*Correspondence:

Herbert Tilg

herbert.tilg@i-med.ac.at

Specialty section:

This article was submitted to

Molecular and Structural

Endocrinology,

a section of the journal

Frontiers in Endocrinology

Received: 12 June 2019

Accepted: 22 August 2019

Published: 06 September 2019

Citation:

Grabherr F, Grander C, Effenberger M,

Adolph TE and Tilg H (2019) Gut

Dysfunction and Non-alcoholic Fatty

Liver Disease.

Front. Endocrinol. 10:611.

doi: 10.3389/fendo.2019.00611
Non-alcoholic fatty liver disease (NAFLD) has emerged as one of the leading liver diseases worldwide. NAFLD is characterized by hepatic steatosis and may progress to an inflammatory condition termed non-alcoholic steatohepatitis (NASH), liver cirrhosis, and hepatocellular carcinoma. It became evident in the last years that NAFLD pathophysiology is complex and involves diverse immunological and metabolic pathways. An association between intestinal signals (e.g., derived from the gut microbiota) and the development of obesity and its metabolic consequences such as NAFLD are increasingly recognized. Pre-clinical studies have shown that germ-free mice are protected against obesity and hepatic steatosis. Several human studies from the past years have demonstrated that NAFLD contains a disease-specific gut microbiome signature. Controlled studies propose that certain bacteria with rather pro-inflammatory features such as Proteobacteria or Escherichia coli are dominantly present in these patients. In contrast, rather protective bacteria such as Faecalibacterium prausnitzii are decreased in NAFLD patients. Furthermore, various bacterial metabolites and microbiota-generated secondary bile acids are involved in NAFLD-associated metabolic dysfunction. Although these findings are exciting, research currently lack evidence that interference at the level of the gut microbiome is beneficial for these diseases. Further preclinical and clinical studies are needed to advance this aspect of NAFLD research and to support the notion that the intestinal microbiota is indeed of major relevance in this disorder.

Keywords: NAFLD, microbiota, inflammation, diabetes, liver

\section{INTRODUCTION}

Non-alcoholic fatty liver disease (NAFLD) has emerged in the last years as the most common liver disease worldwide (1). The reason for this development is mainly based on the observation that obesity and obesity-related disorders such as type 2 diabetes (T2D) are on the rise, and NAFLD reflects a typical complication of these disorders (2). Importantly, hepatic steatosis might evolve toward its inflammatory complication i.e., non-alcoholic steatohepatitis (NASH), liver cirrhosis, and hepatocellular carcinoma $(3,4)$. It is currently believed that $\sim 10-20 \%$ of all subjects with NAFLD develop NASH. Whereas, advances in the NAFLD field have been substantial in the past years, it remains still unclear why certain subjects develop NASH while the majority of patients do not progress. Although it is clear that obesity plays a key role in NAFLD pathogenesis, aspects behind the obesity epidemic remain unresolved and might not simply reflect calorie consumption. It has long been assumed that certain host factors and particularly the gut microbiota is involved in the evolution of this disease toward its inflammatory phenotype i.e., NASH (5). 
The human gastrointestinal tract contains a huge number of bacteria, archaea, and viruses (6-10). The development of modern molecular and sequencing technologies allowed medical research in the last years to get some insights into this exciting bacterial world (11). Recent calculations proposed that three bacterial cells exist for every single cell of the human body (12). Whereas, it has been initially assumed that the genes of the gut microbiome mainly control functions regarding digestion of complex carbohydrates or regulating immune processes, recent evidence indicates that the microbiome has major functions in directing metabolic pathways in health and disease (13). The gut microbiota also controls the integrity of the intestinal epithelial barrier and thereby substantially contributes to gastrointestinal health (14-16) (Figure 1A). Indeed, evidence highlights that mucosal integrity is affected in obesity-related disorders including NAFLD (17).

It is increasingly acknowledged that the intestinal microbiota regulates metabolic functions and plays a role in the pathogenesis of NAFLD. Animal experiments suggested that the gut microbiota is critically involved in development of adipose tissue (18) and also affects evolution of hepatic steatosis (19). Indeed, early human studies found that obese subjects exhibit a gut microbiome signature (20). Several preclinical and clinical studies from the last years have investigated the role of the gut microbiota in fatty liver diseases and demonstrated that indeed a gut microbiome signature might be present in these disorders $(21,22)$. In addition, it seems likely that numerous bidirectional interactions exist between the liver and the gastrointestinal tract where the gut microbiota affects processes in the liver in health and disease and on the other side liver-released mediators such as bile acids control metabolic functions and the intestinal microbiota. We describe in this article recent evidence for a key role of the gut microbiome and related metabolites in the pathophysiology of NAFLD.

\section{BACTERIAL DYSBIOSIS IN NAFLD}

\section{Pre-clinical Studies}

Landmark animal experiments performed by Bäckhed et al. suggested that the gut microbiota affects the development of adipose tissue (18). Equally important studies from Anna Mae Diehl's laboratory showed that the use of a certain probiotic decreased hepatic steatosis to a similar extent than treatment with an anti-TNF antibody (19) suggesting that both microbes and inflammatory pathways contribute to evolution of hepatic steatosis. One of the first convincing experimental studies supporting a key role for the gut microbiota in NAFLD came from a study by Le Roy and colleagues (23). In this study, the authors report that C57BL/6J mice developed similar obesity but responded metabolically differently toward a high fat diet (HFD): one group exhibited hyperglycemia and increased plasma concentrations of pro-inflammatory cytokines whereas the socalled "non-responder" group remained normoglycemic without significant systemic inflammation. In case germ-free mice were colonized with the gut microbiota from both groups, only mice receiving "responder" stool developed hepatic steatosis suggesting that intestinal microbiota allowed to transfer disease phenotype and liver steatosis (23).

Interference at the gut microbiome level by either pre-, pro-, or antibiotics might therefore affect disease phenotype. A prebiotic i.e., guar gum substantially changed the gut microbiota composition in a HFD model, decreased diet-induced obesity, and ameliorated glucose tolerance but interestingly even worsened liver phenotype leading to more inflammation and fibrosis (24). Chronic oral administration of an antibiotic in this model suppressed intestinal bacteria, reduced portal secondary bile acid levels, and decreased liver inflammation and fibrosis suggesting an important link between changes in intestinal microbiota, liver inflammation and fibrosis at least in a pre-clinical model probably via alterations in bile acids (24). Interestingly, manipulation at the level of intestinal microbiota might even improve more advanced NASH (25). In this study, rats were fed a HFD and high glucose/fructose syrup (HFGFD) and exhibited NASH including portal hypertension. Importantly, portal hypertension was improved after fecal transplantation from a healthy rat (25).

Obesity rates are increasing worldwide and maternal obesity confers risk to developing disease in offspring (26). In this recent study, the authors compared germ-free mice colonized with feces from 2-week-old infants born to obese or normalweight mothers. Mice exposed to stool from infants born to obese mothers demonstrated enhanced liver gene expression for pathways such as endoplasmic reticulum stress and innate immunity combined with histological evidence of periportal inflammation. In addition, these mice exhibited increased intestinal permeability. When mice were challenged by Westernstyle diet, these mice showed excess weight gain and accelerated NAFLD. This study demonstrated an important interaction between motherhood obesity, infant dysbiosis and childhood obesity and NAFLD (26). From all these preclinical studies we can conclude that evidence for a role of the gut microbiota in NAFLD and related phenotypes is accumulating.

\section{Clinical Evidence for Dysbiosis in NAFLD}

Substantial evidence from the past years suggests that the gut microbiota plays a role in NAFLD. In a clinical study including 22 subjects with NASH and 23 control patients, subjects with NASH showed small intestinal bacterial overgrowth (Figure 1B) and increased circulating endotoxin and $\mathrm{TNF} \alpha$ levels (28). A meta-analysis of five clinical trials including 128 NAFLD patients and 83 control subjects revealed impaired intestinal permeability. These changes were even higher in the subgroup of NASH patients (29). A remarkable case report described a patient with small intestinal bacterial overgrowth, which evolved after jejuno-colic bypass surgery and was reversed after surgical correction (30). NASH patients exhibit a higher prevalence of SIBO and this has been linked with an increased expression of TLR4 on CD14 positive monocytes and higher plasma IL8 levels (31). First reports assessing the gut microbiome in NAFLD patients were presented a few years ago $(32,33)$. In several studies, differences were abundant at phylum, family, and genus levels between healthy controls and NASH subjects (Table 1). Importantly, fewer differences in their microbiome 


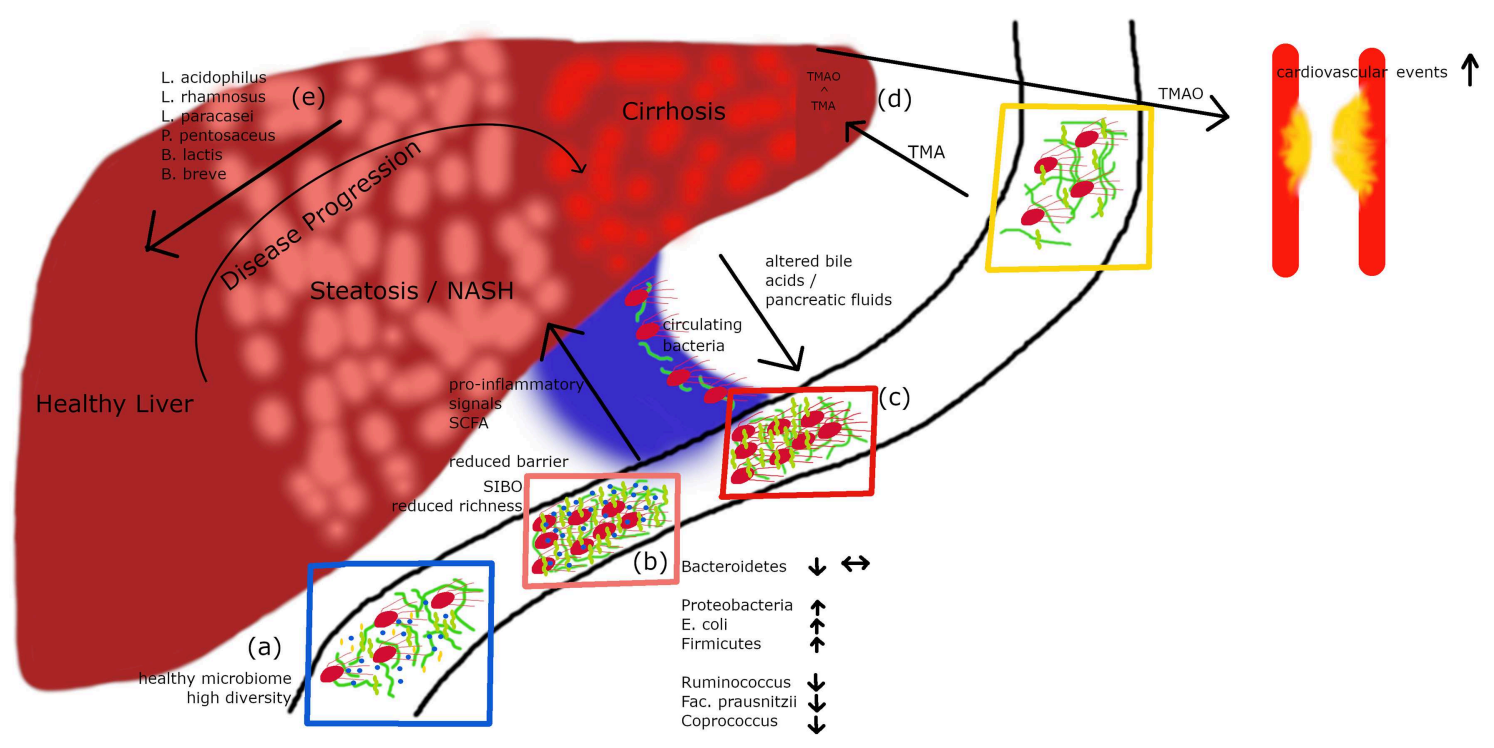

FIGURE 1 | A healthy microbiome is characterized by high diversity of microbial species (A). In patients with hepatic steatosis or NASH a small intestinal overgrowth develops and at the same time the diversity is decreasing (B). Following impaired intestinal barrier function a rise in pro-inflammatory and pro-steatotic bacterial products in the portal circuit may occur, which can drive inflammation/steatosis and hepatic disease. Altered bile acids and pancreatic fluids can influence the microbiome. In cirrhotic patients, culturable bacteria can translocate via the portal vein to the systemic circulation (C). Metabolites derived from the microbiome do not only influence the liver but also other organs. TMA is produced by bacteria out of dietary compounds, in the liver it is further metabolized to TMAO, which leads to an increased risk for cardiovascular events (D). Recently published data (27) show that a cocktail of prebiotics can reduce the intrahepatic fat content in steatotic patients (E).

were observed between obese and NASH subjects. Proteobacteria, Enterobacteriaceae, and Escherichia were the only phylum, family and genus types showing significant differences between obese and NASH patients (32). Another study demonstrated that patients with NASH had a lower rate of Bacteroidetes compared to steatosis and healthy controls (33). This data implicate that there exists an inverse association between the presence of NASH and Bacteroidetes content in feces. In a pediatric NAFLD study, Actinobacteria were increased whereas Bacteroidetes were reduced (34). Furthermore, at species levels Oscillobacter was lower in NAFLD whereas Ruminococcus, Blautia, and Dorea were increased in NASH (34). A further study assessed the gut microbiota and severity of histology-proven NAFLD in 57 patients (35). Here, Bacteroides abundance was enhanced and correlated with disease severity, whereas Prevotella abundance was decreased. In this study Ruminococcus abundance increased in more severe disease especially in advanced fibrosis. In conclusion, these studies underline that NASH is associated with a "microbiome signature" which could promote disease progression and the clinical phenotype.

Interestingly, Schierwagen et al. recently detected a circulating microbiome when studying central, hepatic and portal venous blood and peripheral blood from seven liver cirrhosis patients receiving a transjugular portosystemic shunt (37) (Figure 1C). Proteobacteria dominated in the circulation and changes were compartment-specific. Interestingly, in a small group of patients the investigators were able to cultivate respective bacteria suggesting that detected bacteria are viable and potentially bioactive. They also observed a direct correlation with cytokine
TABLE 1 | Overview of intestinal microbiota changes in NAFLD.

\begin{tabular}{ll}
\hline Enriched in NAFLD & Bacteroides (Genus) (35) \\
& Ruminococcus (Genus) (35) \\
& Lactobacillaceae (Family) (36) \\
& Lactobacillus (Genus) (36) \\
& Proteobacteria (Phylum) (22) \\
& E. coli (Species) (22) \\
\hline Decreased in NAFLD & Actinobacteria (Phylum) (34) \\
& Bacteroidetes (Phylum) (34) \\
& Oscillobacter (Genus) (34) \\
& Prevotella (Genus) (35) \\
Ruminococcus (Genus) (36) & Faecalibacterium prausnitzii (Species) (36) \\
Coprococcus (Genus) (36) \\
Firmicutes (Phylum) (22)
\end{tabular}

levels arguing that systemic inflammation detected in liver cirrhosis patients might be associated with gut-derived bacteria. A circulatory systemic blood microbiome signature has also been described in a small NAFLD study assessing obese subjects with fibrosis (38), although the fecal microbiome was not studied. Severe alcoholic hepatitis is probably one of the diseases with the most severe impaired epithelial barrier. Another study found that these patients show a microbiome profile with a decrease in Bacteroidetes and an enrichment of Fusobacteria, bacteria which are mainly found in the oral cavity (39). This 
phenomenon was accompanied by higher endotoxemia and activation of type II secretion which has been related with the virulence of gram negative bacteria (39). Although these first studies open an exciting avenue, many more careful studies are needed to prove a relevance for "systemically" detectable microbiome.

The most convincing evidence for a potential role of the intestinal microbiota in human NAFLD has been reported by Loomba et al. (22). In this study, which assessed 86 patients with biopsy-proven NAFLD, the authors characterized 37 different bacteria which enabled to define mild vs. advanced fibrosis. Advanced fibrosis was characterized by an increased abundance of Proteobacteria and E.coli and a decrease in Firmicutes supporting the notion that a panel of microbiome markers potentially enables to diagnose advanced fibrosis in NAFLD. The severity of liver disease in NAFLD also might correlate with microbiome patterns (35). Bacteroides concentration was increased dependent on severity of disease, whereas Prevotella abundance decreased. Ruminococcus increased in more severe disease if advanced fibrosis was present (Table 1). Loomba's group recently reported a gut microbiome signature specific for NAFLD cirrhosis (21). In this study, they found that a panel of 30 features, including a panel of 27 bacteria, discriminated NAFLDcirrhosis in a random forest classifier model. They strengthened their data by including both a derivation and validation cohort showing similar results (21). Some evidence has been reported that there is an association between a disturbed gut microbiome in NAFLD independent from the presence of obesity or insulin resistance (36). In this prospective cross-sectional study 39 adults with biopsy-proven NAFLD (15 simple steatosis, $24 \mathrm{NASH}$, and 28 healthy controls) were investigated. In NAFLD, Lactobacillus and Lactobacillaceae were more abundant compared to healthy controls. Lower abundance in both NAFLD patients compared to healthy controls were confirmed by qPCR for Ruminococcus, Faecalibacterium prausnitzii, and Coprococcus. Importantly, the lower abundance in NAFLD was independent of body weight and insulin resistance (36). Especially findings on Ruminococcus are rather controversial, as two other studies showed an increase in its presence in NAFLD suggesting that further studies are needed $(34,35)$. Only very few interventional studies in human NAFLD are so far available (27). This study assessed the effects of a probiotic treatment consisting of Lactobacillus acidophilus, L. rhamnosus, L. paracasei, Pediococcus pentosaceus, Bifidobacterium lactis, and $B$. breve on visceral and intrahepatic fat in NAFLD. Sixty-eight obese NAFLD patients were treated with either a probiotic or placebo for 12 weeks. Interestingly, body weight and total body fat decreased in the probiotic group but not in the placebo group. Treatment with this probiotic for 12 weeks decreased intrahepatic fat and body weight in obese NAFLD patients (Figure 1E) (27). Overall, evidence is evolving that there exists similar to obesity (40) and type 2 diabetes (41) a "gut microbiotal signature" in NAFLD which might allow us in the future to differentiate between patients with simply fatty liver and NASH and might furthermore allow to elucidate underlying pathomechanisms in the development of NASH. This also holds the promise that manipulation at this level might improve disease phenotype.

\section{WHAT CAN WE LEARN FROM MICROBIOME STUDIES IN TYPE 2 DIABETES?}

Several studies from the past years have suggested that a gut microbiome signature exists in diabetes $(42,43)$. Interestingly, in certain studies bacterial strains could be defined which are potentially involved in the regulation of certain features of T2D such as insulin resistance. Pedersen et al. found that the human intestinal microbiome affects the serum metabolome exhibiting increased levels of branched-chain amino acids (BCAA) and this was associated with insulin resistance in non-diabetic subjects (44). Prevotella copri and Bacteroidis vulgatus, two bacterial strains being identified in T2D subjects, caused insulin resistance in murines and upregulated levels of BCAA in these animals (44). Bacterial DNA was detected in mesenteric adipose tissue suggesting an impaired intestinal barrier and in another study in T2D patients Ralstonia pickettii was one of the most prevalent bacterial strains (45). The intestinal epithelium plays an important role in health and metabolic functions and an exciting recent study demonstrated that glucose is able to affect intestinal mucosal integrity (46). Certain bacterial strains such as Eubacterium hallii might be beneficial and are able to improve insulin resistance in rodent experiments (47). E.hallii treatment improved energy expenditure, enhanced fecal concentrations of butyrate, and affected bile acid metabolism. Similar to the Pedersen study (44), a recent report also observed that patients with pre-diabetes exhibit an aberrant intestinal microbiota accompanied by a decrease of A.muciniphila (48).

What comes first remains a key question. Hyperglycemia impairs epithelial integrity (46) and could exhibit a relevant factor influencing composition of the intestinal microbiota. In addition, an altered microbiota early in disease evolution of T2D could subsequently affect diverse disease characteristics of diabetes such as insulin resistance. Studies from pre-diabetes suggest that dysbiosis might reflect a rather early change in this disease (44, 48). Additional studies investigating well-characterized patients taking care of many potential additional factors such as dietary factors, drugs, or stool consistency are awaited to further strengthen the association of an altered intestinal microbiota with diabetes. The final proof, however, for a key role of the intestinal microbiota in type 2 diabetes depends on clinical trials where such new identified bacteria when administered to patients will demonstrate beneficial metabolic effects.

\section{ROLE OF BACTERIAL METABOLITES IN NAFLD}

The gut microbiota contributes substantially to circulating metabolites in the human body. These metabolites may act proinflammatory, direct metabolic processes, act protective, and anti-inflammatory or as probably true for most cases exert rather unknown biological functions. A recent chemical genetic screen 
revealed that many bacterial members of the gut microbiota release metabolites commonly interacting with so called Gprotein coupled receptors (GPCRs) (49). This is potentially of major biological relevance as GPCRs are expressed by many cell types throughout the body and exert mainly immune and metabolic functions $(50,51)$.

Some metabolites, such as short chain fatty acids (SCFA) acetate, butyrate, or propionate can only be produced by bacterial fermentation, that is digestion of complex carbohydrates. Fecal SCFAs concentrations correlate with the presence of certain bacteria such as Alistipes, Barnesiella, Prevotella, or inversely with Bacteroides at least in mice (52). SCFAs are metabolically highly active, mainly act via GPCRs, such as Gpr41 with its ligand propionate (53). Importantly, Gpr41-/- mice do not exhibit obesity despite high calorie challenge (54). The exact mechanisms of SCFAs in NAFLD are incompletely understood. Some evidence suggests that SCFAs promote gluconeogenesis and lipogenesis (55) which is also supported by clinical evidence (56). In contrast, many studies in the field of metabolism suggest that they act metabolically beneficial $(57,58)$. Butyrate is also suppressing HFD-induced obesity and insulin resistance (59). Another protective mechanism of SCFAs might be mediated via blocking histone deacetylases (HDACs) and thereby inducing regulatory $\mathrm{T}$ cells which could reduce insulin resistance (60-64).

Dietary factors such as phopsphatidylcholine, choline and carnitine are metabolized by the gut microbiota to generate trimethylamine (TMA) which is converted in the liver to TMA-N-oxide (TMAO) by flavin-containing monoxygenases. TMAO levels significantly relate with atherosclerosis and associated cardiovascular complications such as myocardial infarction and stroke as this metabolite promotes inflammation and platelet activation $(65,66)$ (Figure 1D). Increased TMAO levels correlate with the presence of T2D, glycemic control in T2D, its complications and also NAFLD (67-70).

A large study assessed the relationship between metabolome, the gut microbiome and hepatic transcriptome (71). Amount of hepatic steatosis was accompanied by a certain microbiome signature defined by decreased microbial gene richness (MGR), and increased rates of Proteobacteria, Actinobacteria, and Verrucomicrobia. A reduced MGR also correlated with an increase in branched chain amino acids (BCAA) pool and one bacteria-derived metabolite i.e., phenylacetate was highly associated with steatosis. Phenylacetate, a degradation product of certain essential amino acids such as phenylalanine and tyrosine and possibly other related metabolites enhance hepatic lipid accumulation by increasing BCAA utilization. Interestingly, administration of phenylacetate to mice resulted also in hepatic steatosis although underlying mechanisms are currently not understood.

Interestingly, some metabolites may interact with key metabolic pathways without affecting immune pathways or inflammation. Koh et al. recently identified such a metabolite by characterizing the bacterial metabolite imidazole propionate as a gut-derived factor regulating insulin signaling (72). The authors analyzed portal vein blood from patients with T2D for potential metabolically active factors. When the authors finally characterized imidazole propionate as a microbially produced histidine-derived metabolite they observed that subjects with T2D not only had higher circulating concentrations of this metabolite but also observed that imidazole propionate affected insulin signaling by activation of p38 $\gamma$ MAPK and phosphorylation of p62 which finally resulted in activation of mechanistic target of rapamycin (mTORC1). This study nicely illustrates how bacterial metabolites besides directing key inflammatory pathways might also control other key pathways of metabolic dysfunction (72). The here discussed metabolites reflect only some recently characterized microbiota-driven or microbiotaderived metabolites highlighting the enormous potential of this rather novel field.

\section{NAFLD: A PROTOTYPIC SYSTEMIC INFLAMMATORY DISEASE}

Low-grade inflammation is a hallmark of many chronic disorders such as chronic liver diseases or T2D and it remains currently unclear which compartments in the body might contribute to this phenomenon. Several NAFLD studies have shown increased circulating inflammatory parameters including cytokines, acute phase proteins, and adhesion molecules especially in NASH patients (73-76). Detectable systemic inflammation in these metabolic "non-communicable" disorders is usually considered sterile and driven mainly by innate immune factors $(77,78)$. Recent data from microbiome research, however, suggest that the gastrointestinal tract with its harbored microbiota might reflect an important source of inflammatory mediators and metabolites (5).

Low-grade chronic inflammation is of major relevance for NAFLD patients within and outside the liver. Although NAFLD patients most commonly die because of cardiovascular complications and atherosclerosis $(79,80)$, death due too liver disease is also frequent. Complications of NAFLD such as NASH, liver cirrhosis, and hepatocellular carcinoma are mainly but not solely inflammation-driven. It is now well-accepted that the amount of fibrosis defines the long-term prognosis of NAFLD-associated liver disease $(81,82)$, which is in most instances driven by inflammation. Therefore, inflammation substantially contributes to patient outcome both in (liver cirrhosis) and outside the liver (atherosclerosis, cardiovascular complications) in NAFLD.

Inflammatory events in case of NAFLD also have major metabolic consequences i.e., regulation of insulin signaling and insulin resistance. Indeed, insulin resistance has long been assumed to be controlled by inflammatory pathways (83). Many immune factors especially certain proinflammatory cytokines have been demonstrated to affect insulin signaling in various tissues. These studies are, however, dominated by preclinical reports and final prove in humans for such a role is still somewhat missing. Many studies have assessed the role of innate immune factors and cytokines in insulin 
signaling. We have recently shown, that interleukin 37 (IL37), a human anti-inflammatory cytokine plays an adipokinelike anti-inflammatory and metabolically beneficial role in metabolic dysfunction $(84,85)$. Patients undergoing substantial weight loss consequent to bariatric surgery exhibited a massive increase of subcutaneous adipose tissue IL-37 expression similar as observed for adiponectin (84). When using transgenic IL37 (IL-37tg) mice we observed substantial improvement of metabolic dysfunction in various models of obesity-related disorders including improvement of insulin sensitivity (85). Importantly, we also observed a steady-state IL-37 mRNA expression level in humans that directly correlated with insulin sensitivity and indirectly with degree of inflammation in adipose tissue. Type I interferon signaling induced by innate and adaptive immunity controls inflammatory processes subsequent to infection. The role of type I interferon signaling in metabolic diseases and the development of NAFLD is unclear. We recently assessed the role of type I interferon signaling in tissue-specific type I interferon receptor (IFNAR1) knockout mice (86). Adipose-, but not hepatic tissue-specific deletion of IFNAR1 worsened metabolic functions associated with a HFD characterized by enhanced weight gain, insulin resistance, and an impaired glucose tolerance compared to wild-type mice. Interestingly, deleted type I interferon signaling in myeloid or intestinal-epithelial cells did not modulate susceptibility to metabolic or hepatic disease. This study points out that type I interferon signaling in adipose tissue might play a role by in metabolic disorders such as NAFLD. A detailed discussion of this topic is beyond the scope of this article.

In conclusion, recent data indicates that chronic inflammation possibly derived from the gut microbiota substantially contributes to metabolic disorders such as NAFLD, which is a key event that guides patient outcome.

\section{LIVER-DERIVED SIGNALS AFFECTING THE GUT MICROBIOME: BILE ACIDS AS KEY PLAYERS}

Whereas, life-long regulation of the composition of the intestinal microbiota is crucial for health and extrinsic factors such as dietary components reflect probably the key confounders, it is increasingly recognized that intrinsic factors with close proximity to the gastrointestinal tract such as bile or pancreatic fluid might be of equal importance $(15,87,88)$. Bile acids not only regulate major metabolic pathways via interaction with mainly so-called nuclear receptors but also affect growth and regulation of the intestinal microbiota (15). Bile acids are generated by the liver from cholesterol and metabolized in the intestine by the intestinal microbiota. They signal via the nuclear farnesoid $\mathrm{X}$ receptor (FXR) and the G protein-coupled membrane receptor 5 (TGR5), which control various metabolic and immune pathways in the host.

The primary bile acids cholic acid and chenodeoxycholic acid in humans and muricholic acid in mice are produced in the liver as cholesterol derivates and secreted into the intestine as glycine and taurine conjugates. In the gut, these secondary bile acids interact with various nuclear receptors such as FXR, TGR5, pregnane X receptor, or vitamin D receptor (89). Recently it has been demonstrated that metformin affects the gut microbiota i.e., decreasing B.fragilis concentrations and in parallel resulted in an increase of the murine bile acid glycoursodeoxycholic acid (GUDCA) resulting in inhibition of intestinal FXR activation and respective metabolic benefits (90). Interestingly FXR activation in rodents was required for postoperative improvements in glycemia whereas changes in body weight, food intake, and other factors were unaffected (91). In this preclinical study bile diversion to the ileum improved glucose homeostasis via the FXR-Glp-1 axis and improvements were paralleled by an increase in intestinal Akkermansia muciniphila concentrations (91). We recently demonstrated that after massive weight loss after bariatric surgery (laparoscopic adjustable gastric banding, LAGB) both conjugated and secondary bile acids increased 3 months after LAGB and only one bile acid i.e., glycolithocholic acid sulfate remained significantly elevated even after 1 year (92). Furthermore, changes in bile acids importantly correlated with Glp-1 and fibroblast growth factor 19 levels supporting the notion that bile acid changes after LAGB crucially affect metabolic pathways. Many more studies have demonstrated in the past years that bile acids and derivates are crucial metabolic players and interference at the level of FXR could indeed reflect a promising treatment strategy for NAFLD in the future (93).

\section{CONCLUSIONS AND OUTLOOK}

We discussed several pathways involved in prototypic gut-liver interactions. While a complex gastrointestinal bacterial world exerts and initiates biological processes throughout the body, underlying mechanisms are poorly understood. Studies from the past years have now convincingly shown that NAFLD patients at various stages of their liver disease have a specific gut microbiome signature. It becomes increasingly recognized that especially gram-negative bacteria such as Enterobacteria, Escherichia coli, and Proteobacteria are increased in concentrations and activities and may contribute to the pro-inflammatory phenotype of this disorder. One bacterial product which seems of key importance is endotoxin, which is elevated in advanced NAFLD $(94,95)$. It is still unclear which intestinal bacteria reflect the major source of this key driver of inflammation.

An equally exciting field is the topic of bacterial metabolites and their relevance in fatty liver diseases. Several excellent studies have proven that such metabolites regulate evolution of hepatic steatosis and insulin signaling. What is also increasingly acknowledged is the fact that gut-liver interactions take place bidirectionally and there exists also a major liver-gut axis meaning that products of the liver affect composition of the gut microbiome. Prototypic factors into this direction are mainly bile acids and especially secondary bile acids, which are again regulated by the gut microbiota. Bile acids and derivates interact and bind to nuclear receptors, which regulate key metabolic pathways involved in NAFLD. From all these studies it becomes evident that the interaction of gut bacteria, immunity and 
metabolic pathways are key to our understanding of this frequent liver disorder. Deciphering disease processes will allow to develop innovative treatment modalities that are desperately needed for NAFLD.

\section{AUTHOR CONTRIBUTIONS}

All authors listed have made a substantial, direct and intellectual contribution to the work, and approved it for publication.

\section{REFERENCES}

1. Diehl AM, Day C. Cause, pathogenesis, and treatment of nonalcoholic steatohepatitis. $N$ Engl $J$ Med. (2017) 377:2063-72. doi: 10.1056/NEJMra1503519

2. Tilg H, Moschen AR, Roden M. NAFLD and diabetes mellitus. Nat Rev Gastroenterol Hepatol. (2017) 14:32-42. doi: 10.1038/nrgastro.2016.147

3. Castera L, Friedrich-Rust M, Loomba R. Noninvasive assessment of liver disease in patients with nonalcoholic fatty liver disease. Gastroenterology. (2019) 156:1264-81.e4. doi: 10.1053/j.gastro.2018.12.036

4. Lackner C, Tiniakos D. Fibrosis and alcohol-related liver disease. J Hepatol. (2019) 70:294-304. doi: 10.1016/j.jhep.2018.12.003

5. Moschen AR, Kaser S, Tilg H. Non-alcoholic steatohepatitis: a microbiota-driven disease. Trends Endocrinol Metab. (2013) 24:537-45. doi: 10.1016/j.tem.2013.05.009

6. Marchesi JR, Adams DH, Fava F, Hermes GD, Hirschfield GM, Hold G, et al. The gut microbiota and host health: a new clinical frontier. Gut. (2016) 65:330-9. doi: 10.1136/gutjnl-2015-309990

7. Lynch SV, Pedersen O. The human intestinal microbiome in health and disease. N Engl J Med. (2016) 375:2369-79. doi: 10.1056/NEJMra1600266

8. Maruvada P, Leone V, Kaplan LM, Chang EB. The human microbiome and obesity: moving beyond associations. Cell Host Microbe. (2017) 22:589-99. doi: 10.1016/j.chom.2017.10.005

9. Costea PI, Hildebrand F, Arumugam M, Backhed F, Blaser MJ, Bushman FD, et al. Enterotypes in the landscape of gut microbial community composition. Nat Microbiol. (2018) 3:8-16. doi: 10.1038/s41564-017-0072-8

10. Cani PD. Severe obesity and gut microbiota: does bariatric surgery really reset the system? Gut. (2019) 68:5-6. doi: 10.1136/gutjnl-2018-316815

11. Pasolli E, Asnicar F, Manara S, Zolfo M, Karcher N, Armanini F, et al. Extensive unexplored human microbiome diversity revealed by over 150,000 genomes from metagenomes spanning age, geography, and lifestyle. Cell. (2019) 176:649-62.e20. doi: 10.1016/j.cell.2019. 01.001

12. Sender R, Fuchs S, Milo R. Are we really vastly outnumbered? Revisiting the ratio of bacterial to host cells in humans. Cell. (2016) 164:337-40. doi: 10.1016/j.cell.2016.01.013

13. Gilbert JA, Blaser MJ, Caporaso JG, Jansson JK, Lynch SV, Knight R. Current understanding of the human microbiome. Nat Med. (2018) 24:392-400. doi: $10.1038 / \mathrm{nm} .4517$

14. Bischoff SC, Barbara G, Buurman W, Ockhuizen T, Schulzke JD, Serino M, et al. Intestinal permeability-a new target for disease prevention and therapy. BMC Gastroenterol. (2014) 14:189. doi: 10.1186/s12876-014-0189-7

15. Wahlström A, Sayin SI, Marschall HU, Backhed F. Intestinal crosstalk between bile acids and microbiota and its impact on host metabolism. Cell Metab. (2016) 24:41-50. doi: 10.1016/j.cmet.2016.05.005

16. Sonnenburg JL, Backhed F. Diet-microbiota interactions as moderators of human metabolism. Nature. (2016) 535:56-64. doi: 10.1038/nature 18846

17. Nier A, Engstler AJ, Maier IB, Bergheim I. Markers of intestinal permeability are already altered in early stages of non-alcoholic fatty liver disease: Studies in children. PLoS ONE. (2017) 12:e0183282. doi: 10.1371/journal.pone. 0183282

18. Bäckhed F, Ding H, Wang T, Hooper LV, Koh GY, Nagy A, et al. The gut microbiota as an environmental factor that regulates fat storage. Proc Natl Acad Sci USA. (2004) 101:15718-23. doi: 10.1073/pnas.0407076101

\section{FUNDING}

This work was supported by the excellence initiative VASCage (Centre for Promoting Vascular Health in the Ageing Community), an R\&D K-Centre (COMET program Competence Centers for Excellent Technologies) funded by the Austrian Ministry for Transport, Innovation and Technology, the Austrian Ministry for Digital and Economic Affairs and the federal states Tyrol, Salzburg and Vienna.

19. Li Z, Yang S, Lin H, Huang J, Watkins PA, Moser AB, et al. Probiotics and antibodies to TNF inhibit inflammatory activity and improve nonalcoholic fatty liver disease. Hepatology. (2003) 37:343-50. doi: 10.1053/jhep.2003.50048

20. Turnbaugh PJ, Ley RE, Mahowald MA, Magrini V, Mardis ER, Gordon JI. An obesity-associated gut microbiome with increased capacity for energy harvest. Nature. (2006) 444:1027-31. doi: 10.1038/nature05414

21. Caussy C, Tripathi A, Humphrey G, Bassirian S, Singh S, Faulkner C, et al. A gut microbiome signature for cirrhosis due to nonalcoholic fatty liver disease. Nat Commun. (2019) 10:1406. doi: 10.1038/s41467-019-09455-9

22. Loomba R, Seguritan V, Li W, Long T, Klitgord N, Bhatt A, et al. Gut microbiome-based metagenomic signature for non-invasive detection of advanced fibrosis in human nonalcoholic fatty liver disease. Cell Metab. (2017) 25:1054-1062.e5. doi: 10.1016/j.cmet.2017.04.001

23. Le Roy T, Llopis M, Lepage P, Bruneau A, Rabot S, Bevilacqua C, et al. Intestinal microbiota determines development of non-alcoholic fatty liver disease in mice. Gut. (2013) 62:1787-94. doi: 10.1136/gutjnl-2012-303816

24. Janssen AWF, Houben T, Katiraei S, Dijk W, Boutens L, Van Der Bolt N, et al. Modulation of the gut microbiota impacts nonalcoholic fatty liver disease: a potential role for bile acids. J Lipid Res. (2017) 58:1399-416. doi: 10.1194/jlr.M075713

25. Garcia-Lezana T, Raurell I, Bravo M, Torres-Arauz M, Salcedo MT, Santiago A, et al. Restoration of a healthy intestinal microbiota normalizes portal hypertension in a rat model of nonalcoholic steatohepatitis. Hepatology. (2018) 67:1485-98. doi: 10.1002/hep.29646

26. Soderborg TK, Clark SE, Mulligan CE, Janssen RC, Babcock L, Ir $\mathrm{D}$, et al. The gut microbiota in infants of obese mothers increases inflammation and susceptibility to NAFLD. Nat Commun. (2018) 9:4462. doi: 10.1038/s41467-018-06929-0

27. Ahn SB, Jun DW, Kang BK, Lim JH, Lim S, Chung MJ. Randomized, double-blind, placebo-controlled study of a multispecies probiotic mixture in nonalcoholic fatty liver disease. Sci Rep. (2019) 9:5688. doi: 10.1038/s41598-019-42059-3

28. Wigg AJ, Roberts-Thomson IC, Dymock RB, Mccarthy PJ, Grose RH, Cummins AG. The role of small intestinal bacterial overgrowth, intestinal permeability, endotoxaemia, and tumour necrosis factor alpha in the pathogenesis of non-alcoholic steatohepatitis. Gut. (2001) 48:206-11. doi: 10.1136/gut.48.2.206

29. Luther J, Garber JJ, Khalili H, Dave M, Bale SS, Jindal R, et al. Hepatic injury in nonalcoholic steatohepatitis contributes to altered intestinal permeability. Cell Mol Gastroenterol Hepatol. (2015) 1:222-32. doi: 10.1016/j.jcmgh.2015.01.001

30. Sung YK, Gwak GY, Choi MS, Koh KC, Paik SW, Yoo BC, et al. A case of nonalcoholic steatohepatitis and small intestinal bacterial overgrowth with peripheral edema caused by intestinal bypass surgery and relieved by repair. Gut Liver. (2012) 6:520-3. doi: 10.5009/gnl.2012.6.4.520

31. Shanab AA, Scully P, Crosbie O, Buckley M, O'mahony L, Shanahan F, et al. Small intestinal bacterial overgrowth in nonalcoholic steatohepatitis: association with toll-like receptor 4 expression and plasma levels of interleukin 8. Dig Dis Sci. (2011) 56:1524-34. doi: 10.1007/s10620-010-1447-3

32. Zhu L, Baker SS, Gill C, Liu W, Alkhouri R, Baker RD, et al. Characterization of gut microbiomes in nonalcoholic steatohepatitis (NASH) patients: a connection between endogenous alcohol and NASH. Hepatology. (2013) 57:601-9. doi: 10.1002/hep.26093

33. Mouzaki M, Comelli EM, Arendt BM, Bonengel J, Fung SK, Fischer SE, et al. Intestinal microbiota in patients with nonalcoholic fatty liver disease. Hepatology. (2013) 58:120-7. doi: 10.1002/hep.26319 
34. Del Chierico F, Nobili V, Vernocchi P, Russo A, Stefanis C, Gnani D, et al. Gut microbiota profiling of pediatric nonalcoholic fatty liver disease and obese patients unveiled by an integrated meta-omics-based approach. Hepatology. (2017) 65:451-64. doi: 10.1002/hep.28572

35. Boursier J, Mueller O, Barret M, Machado M, Fizanne L, Araujo-Perez F, et al. The severity of nonalcoholic fatty liver disease is associated with gut dysbiosis and shift in the metabolic function of the gut microbiota. Hepatology. (2016) 63:764-75. doi: 10.1002/hep.28356

36. Da Silva HE, Teterina A, Comelli EM, Taibi A, Arendt BM, Fischer SE, et al. Nonalcoholic fatty liver disease is associated with dysbiosis independent of body mass index and insulin resistance. Sci Rep. (2018) 8:1466. doi: 10.1038/s41598-018-19753-9

37. Schierwagen R, Alvarez-Silva C, Madsen MSA, Kolbe CC, Meyer C, Thomas D, et al. Circulating microbiome in blood of different circulatory compartments. Gut. (2019) 68:578-80. doi: 10.1136/gutjnl-2018-316227

38. Lelouvier B, Servant F, Paisse S, Brunet AC, Benyahya S, Serino $\mathrm{M}$, et al. Changes in blood microbiota profiles associated with liver fibrosis in obese patients: a pilot analysis. Hepatology. (2016) 64:2015-27. doi: 10.1002/hep.28829

39. Puri P, Daita K, Joyce A, Mirshahi F, Santhekadur PK, Cazanave S, et al. The presence and severity of nonalcoholic steatohepatitis is associated with specific changes in circulating bile acids. Hepatology. (2018) 67:534-48. doi: 10.1002/hep.29359

40. Stanislawski MA, Dabelea D, Wagner BD, Iszatt N, Dahl C, Sontag MK, et al. Gut microbiota in the first 2 years of life and the association with body mass index at age 12 in a Norwegian birth cohort. MBio. (2018) 9:e01751-18. doi: $10.1128 / \mathrm{mBio} .01751-18$

41. Hartstra AV, Bouter KE, Backhed F, Nieuwdorp M. Insights into the role of the microbiome in obesity and type 2 diabetes. Diabetes Care. (2015) 38:159-65. doi: 10.2337/dc14-0769

42. Qin J, Li Y, Cai Z, Li S, Zhu J, Zhang F, et al. A metagenome-wide association study of gut microbiota in type 2 diabetes. Nature. (2012) 490:55-60. doi: $10.1038 /$ nature 11450

43. Karlsson FH, Tremaroli V, Nookaew I, Bergstrom G, Behre CJ, Fagerberg B, et al. Gut metagenome in European women with normal, impaired and diabetic glucose control. Nature. (2013) 498:99-103. doi: 10.1038/nature12198

44. Pedersen HK, Gudmundsdottir V, Nielsen HB, Hyotylainen T, Nielsen T, Jensen BA, et al. Human gut microbes impact host serum metabolome and insulin sensitivity. Nature. (2016) 535:376-81. doi: 10.1038/nature18646

45. Udayappan SD, Kovatcheva-Datchary P, Bakker GJ, Havik SR, Herrema H, Cani PD, et al. Intestinal Ralstonia pickettii augments glucose intolerance in obesity. PLoS ONE. (2017) 12:e0181693. doi: 10.1371/journal.pone.0181693

46. Thaiss CA, Levy M, Grosheva I, Zheng D, Soffer E, Blacher E, et al. Hyperglycemia drives intestinal barrier dysfunction and risk for enteric infection. Science. (2018) 359:1376-83. doi: 10.1126/science.aar3318

47. Udayappan S, Manneras-Holm L, Chaplin-Scott A, Belzer C, Herrema H, Dallinga-Thie GM, et al. Oral treatment with Eubacterium hallii improves insulin sensitivity in db/db mice. NPJ Biofilms Microbiomes. (2016) 2:16009. doi: 10.1038/npjbiofilms.2016.9

48. Allin KH, Tremaroli V, Caesar R, Jensen BAH, Damgaard MTF, Bahl MI, et al. Aberrant intestinal microbiota in individuals with prediabetes. Diabetologia. (2018) 61:810-20. doi: 10.1007/s00125-018-4550-1

49. Chen H, Nwe PK, Yang Y, Rosen CE, Bielecka AA, Kuchroo M, et al. A forward chemical genetic screen reveals gut microbiota metabolites that modulate host physiology. Cell. (2019) 177:1217-1231.e18. doi: 10.1016/j.cell.2019.03.036

50. Cohen LJ, Esterhazy D, Kim SH, Lemetre C, Aguilar RR, Gordon EA, et al. Commensal bacteria make GPCR ligands that mimic human signalling molecules. Nature. (2017) 549:48-53. doi: 10.1038/nature23874

51. Wacker D, Stevens RC, Roth BL. How Ligands Illuminate GPCR Molecular Pharmacology. Cell. (2017) 170:414-27. doi: 10.1016/j.cell.2017.07.009

52. Zhao Y, Wu J, Li JV, Zhou NY, Tang H, Wang Y. Gut microbiota composition modifies fecal metabolic profiles in mice. J Proteome Res. (2013) 12:2987-99. doi: $10.1021 / \mathrm{pr} 400263 \mathrm{n}$

53. Brown AJ, Goldsworthy SM, Barnes AA, Eilert MM, Tcheang L, Daniels D, et al. The Orphan G protein-coupled receptors GPR41 and GPR43 are activated by propionate and other short chain carboxylic acids. J Biol Chem. (2003) 278:11312-9. doi: 10.1074/jbc.M211609200
54. Bellahcene M, O'dowd JF, Wargent ET, Zaibi MS, Hislop DC, Ngala RA, et al. Male mice that lack the G-protein-coupled receptor GPR41 have low energy expenditure and increased body fat content. Br J Nutr. (2013) 109:1755-64. doi: $10.1017 /$ S0007114512003923

55. Weidemann MJ, Hems R, Williams DL, Spray GH, Krebs HA. Gluconeogenesis from propionate in kidney and liver of the vitamin B12-deficient rat. Biochem J. (1970) 117:177-81. doi: 10.1042/bj1170177

56. Rau M, Rehman A, Dittrich M, Groen AK, Hermanns HM, Seyfried $\mathrm{F}$, et al. Fecal SCFAs and SCFA-producing bacteria in gut microbiome of human NAFLD as a putative link to systemic T-cell activation and advanced disease. United European Gastroenterol J. (2018) 6:1496-507. doi: 10.1177/2050640618804444

57. Jin CJ, Sellmann C, Engstler AJ, Ziegenhardt D, Bergheim I. Supplementation of sodium butyrate protects mice from the development of nonalcoholic steatohepatitis (NASH). Br J Nutr. (2015) 114:1745-55. doi: 10.1017/S0007114515003621

58. Mattace Raso G, Simeoli R, Russo R, Iacono A, Santoro A, Paciello $\mathrm{O}$, et al. Effects of sodium butyrate and its synthetic amide derivative on liver inflammation and glucose tolerance in an animal model of steatosis induced by high fat diet. PLoS One. (2013) 8:e68626. doi: 10.1371/journal.pone.0068626

59. Gao Z, Yin J, Zhang J, Ward RE, Martin RJ, Lefevre M, et al. Butyrate improves insulin sensitivity and increases energy expenditure in mice. Diabetes. (2009) 58:1509-17. doi: 10.2337/db08-1637

60. Tao R, De Zoeten EF, Ozkaynak E, Chen C, Wang L, Porrett PM, et al. Deacetylase inhibition promotes the generation and function of regulatory $\mathrm{T}$ cells. Nat Med. (2007) 13:1299-307. doi: 10.1038/nm1652

61. Li H, Gao Z, Zhang J, Ye X, Xu A, Ye J, et al. Sodium butyrate stimulates expression of fibroblast growth factor 21 in liver by inhibition of histone deacetylase 3. Diabetes. (2012) 61:797-806. doi: 10.2337/db11-0846

62. Feuerer M, Herrero L, Cipolletta D, Naaz A, Wong J, Nayer A, et al. Lean, but not obese, fat is enriched for a unique population of regulatory $\mathrm{T}$ cells that affect metabolic parameters. Nat Med. (2009) 15:930-9. doi: 10.1038/nm.2002

63. Cipolletta D, Feuerer M, Li A, Kamei N, Lee J, Shoelson SE, et al. PPAR-gamma is a major driver of the accumulation and phenotype of adipose tissue Treg cells. Nature. (2012) 486:549-53. doi: 10.1038/nature11132

64. Ilan Y, Maron R, Tukpah AM, Maioli TU, Murugaiyan G, Yang K, et al. Induction of regulatory $\mathrm{T}$ cells decreases adipose inflammation and alleviates insulin resistance in ob/ob mice. Proc Natl Acad Sci USA. (2010) 107:9765-70. doi: 10.1073/pnas.0908771107

65. Tang WH, Wang Z, Levison BS, Koeth RA, Britt EB, Fu X, et al. Intestinal microbial metabolism of phosphatidylcholine and cardiovascular risk. $N$ Engl J Med. (2013) 368:1575-84. doi: 10.1056/NEJMoa1109400

66. Zhu W, Gregory JC, Org E, Buffa JA, Gupta N, Wang Z, et al. Gut microbial metabolite TMAO enhances platelet hyperreactivity and thrombosis risk. Cell. (2016) 165:111-24. doi: 10.1016/j.cell.2016.02.011

67. Shan Z, Sun T, Huang H, Chen S, Chen L, Luo C, et al. Association between microbiota-dependent metabolite trimethylamine- $\mathrm{N}$-oxide and type 2 diabetes. Am J Clin Nutr. (2017) 106:888-94. doi: 10.3945/ajcn.117. 157107

68. Tang WH, Wang Z, Li XS, Fan Y, Li DS, Wu Y, et al. Increased trimethylamine $\mathrm{N}$-oxide portends high mortality risk independent of glycemic control in patients with type 2 diabetes mellitus. Clin Chem. (2017) 63:297-306. doi: 10.1373/clinchem.2016.263640

69. Li P, Zhong C, Li S, Sun T, Huang H, Chen X, et al. Plasma concentration of trimethylamine-N-oxide and risk of gestational diabetes mellitus. Am J Clin Nutr. (2018) 108:603-10. doi: 10.1093/ajcn/nqy116

70. Chen YM, Liu Y, Zhou RF, Chen XL, Wang C, Tan XY, et al. Associations of gut-flora-dependent metabolite trimethylamine-N-oxide, betaine and choline with non-alcoholic fatty liver disease in adults. Sci Rep. (2016) 6:19076. doi: $10.1038 /$ srep 19076

71. Hoyles L, Fernandez-Real JM, Federici M, Serino M, Abbott J, Charpentier J, et al. Molecular phenomics and metagenomics of hepatic steatosis in non-diabetic obese women. Nat Med. (2018) 24:1070-80. doi: 10.1038/s41591-018-0061-3

72. Koh A, Molinaro A, Stahlman M, Khan MT, Schmidt C, MannerasHolm L, et al. Microbially produced imidazole propionate impairs 
insulin signaling through mTORC1. Cell. (2018) 175:947-61.e17. doi: 10.1016/j.cell.2018.09.055

73. Haukeland JW, Damas JK, Konopski Z, Loberg EM, Haaland T, Goverud I, et al. Systemic inflammation in nonalcoholic fatty liver disease is characterized by elevated levels of CCL2. J Hepatol. (2006) 44:1167-74. doi: 10.1016/j.jhep.2006.02.011

74. Chiang CH, Huang CC, Chan WL, Chen JW, Leu HB. The severity of non-alcoholic fatty liver disease correlates with high sensitivity Creactive protein value and is independently associated with increased cardiovascular risk in healthy population. Clin Biochem. (2010) 43:1399-404. doi: 10.1016/j.clinbiochem.2010.09.003

75. Zimmermann E, Anty R, Tordjman J, Verrijken A, Gual P, Tran A, et al. C-reactive protein levels in relation to various features of nonalcoholic fatty liver disease among obese patients. J Hepatol. (2011) 55:660-5. doi: 10.1016/j.jhep.2010.12.017

76. Pihlajamäki J, Kuulasmaa T, Kaminska D, Simonen M, Karja V, Gronlund $\mathrm{S}$, et al. Serum interleukin 1 receptor antagonist as an independent marker of non-alcoholic steatohepatitis in humans. J Hepatol. (2012) 56:663-70. doi: $10.1016 /$ j.jhep.2011.10.005

77. Netea MG, Balkwill F, Chonchol M, Cominelli F, Donath MY, GiamarellosBourboulis EJ, et al. A guiding map for inflammation. Nat Immunol. (2017) 18:826-31. doi: $10.1038 /$ ni.3790

78. Kubes P, Mehal WZ. Sterile inflammation in the liver. Gastroenterology. (2012) 143:1158-72. doi: 10.1053/j.gastro.2012.09.008

79. Targher G, Day CP, Bonora E. Risk of cardiovascular disease in patients with nonalcoholic fatty liver disease. $N$ Engl J Med. (2010) 363:1341-50. doi: 10.1056/NEJMra0912063

80. Adams LA, Anstee QM, Tilg H, Targher G. Non-alcoholic fatty liver disease and its relationship with cardiovascular disease and other extrahepatic diseases. Gut. (2017) 66:1138-53. doi: 10.1136/gutjnl-2017-313884

81. Angulo P, Machado MV, Diehl AM. Fibrosis in nonalcoholic Fatty liver disease: mechanisms and clinical implications. Semin Liver Dis. (2015) 35:132-45. doi: 10.1055/s-0035-1550065

82. Angulo P, Kleiner DE, Dam-Larsen S, Adams LA, Bjornsson ES, Charatcharoenwitthaya $\mathrm{P}$, et al. Liver fibrosis, but no other histologic features, is associated with long-term outcomes of patients with nonalcoholic fatty liver disease. Gastroenterology. (2015) 149:389-97.e10. doi: 10.1053/j.gastro.2015.04.043

83. Saltiel AR, Olefsky JM. Inflammatory mechanisms linking obesity and metabolic disease. J Clin Invest. (2017) 127:1-4. doi: 10.1172/JCI92035

84. Moschen AR, Molnar C, Enrich B, Geiger S, Ebenbichler CF, Tilg H. Adipose and liver expression of interleukin (IL)-1 family members in morbid obesity and effects of weight loss. Mol Med. (2011) 17:840-5. doi: 10.2119/molmed.2010.00108

85. Ballak DB, Van Diepen JA, Moschen AR, Jansen HJ, Hijmans A, Groenhof GJ, et al. IL-37 protects against obesity-induced inflammation and insulin resistance. Nat Commun. (2014) 5:4711. doi: 10.1038/ncomms5711
86. Wieser V, Adolph TE, Grander C, Grabherr F, Enrich B, Moser P, et al. Adipose type I interferon signalling protects against metabolic dysfunction. Gut. (2018) 67:157-65. doi: 10.1136/gutjnl-2016-313155

87. Wiest R, Albillos A, Trauner M, Bajaj JS, Jalan R. Targeting the gut-liver axis in liver disease. J Hepatol. (2017) 67:1084-103. doi: 10.1016/j.jhep.2017. 05.007

88. Ahuja M, Schwartz DM, Tandon M, Son A, Zeng M, Swaim W, et al. Orail-mediated antimicrobial secretion from pancreatic acini shapes the gut microbiome and regulates gut innate immunity. Cell Metab. (2017) 25:635-46. doi: 10.1016/j.cmet.2017.02.007

89. De Aguiar Vallim TQ, Tarling EJ, Edwards PA. Pleiotropic roles of bile acids in metabolism. Cell Metab. (2013) 17:657-69. doi: 10.1016/j.cmet.2013.03.013

90. Sun L, Xie C, Wang G, Wu Y, Wu Q, Wang X, et al. Gut microbiota and intestinal FXR mediate the clinical benefits of metformin. Nat Med. (2018) 24:1919-29. doi: 10.1038/s41591-018-0222-4

91. Albaugh VL, Banan B, Antoun J, Xiong Y, Guo Y, Ping J, et al. Role of bile acids and GLP-1 in mediating the metabolic improvements of bariatric surgery. Gastroenterology. (2019) 156:1041-1051.e4. doi: 10.1053/j.gastro.2018. 11.017

92. Thöni V, Pfister A, Melmer A, Enrich B, Salzmann K, Kaser S, et al. Dynamics of bile acid profiles, GLP-1, and FGF19 after laparoscopic gastric banding. J Clin Endocrinol Metab. (2017) 102:2974-84. doi: 10.1210/jc.201700235

93. Mudaliar S, Henry RR, Sanyal AJ, Morrow L, Marschall HU, Kipnes M, et al. Efficacy and safety of the farnesoid $\mathrm{X}$ receptor agonist obeticholic acid in patients with type 2 diabetes and nonalcoholic fatty liver disease. Gastroenterology. (2013) 145:574-82.e1. doi: 10.1053/j.gastro.2013.05.042

94. Fukui H, Brauner B, Bode JC, Bode C. Plasma endotoxin concentrations in patients with alcoholic and non-alcoholic liver disease: reevaluation with an improved chromogenic assay. J Hepatol. (1991) 12:162-9. doi: 10.1016/0168-8278(91)90933-3

95. Miele L, Valenza V, La Torre G, Montalto M, Cammarota G, Ricci R, et al. Increased intestinal permeability and tight junction alterations in nonalcoholic fatty liver disease. Hepatology. (2009) 49:1877-87. doi: 10.1002/hep. 22848

Conflict of Interest Statement: The authors declare that the research was conducted in the absence of any commercial or financial relationships that could be construed as a potential conflict of interest.

Copyright $\odot 2019$ Grabherr, Grander, Effenberger, Adolph and Tilg. This is an openaccess article distributed under the terms of the Creative Commons Attribution License (CC BY). The use, distribution or reproduction in other forums is permitted, provided the original author(s) and the copyright owner(s) are credited and that the original publication in this journal is cited, in accordance with accepted academic practice. No use, distribution or reproduction is permitted which does not comply with these terms. 